\title{
OSCILLATION PROPERTIES OF $y^{n}+p y=0$
}

\author{
GARY D. JONES
}

\begin{abstract}
The purpose of this paper is to give necessary and sufficient conditions for $(k, n-k)$ disconjugacy of $y^{n}+p y=0$. The results are then applied to give counterexamples to the following theorems of Nehari.

If $n$ is even and $p$ is positive either all solutions of $y^{n}+p y=0$ oscillate or none do.

If $n$ is even and $p$ is negative and $y^{n}+p y=0$ has an oscillatory solution then all positive nonoscillatory solutions are either strongly increasing or strongly decreasing.
\end{abstract}

1. Introduction. In [7] Nehari studies the differential equation

$$
y^{(n)}+p y=0
$$

where $n$ is even and $p(x)$ is of constant sign. He reports that nonoscillation of (1) implies ultimate disconjugacy. Elias [1] has given an independent proof which includes the case where $\boldsymbol{n}$ is odd. In Nehari's proof he assumes falsely, as can be seen by examples contained herein, that

$$
\int_{t}^{\infty}(s-t)^{n-k+1} p(s) u(s) d s<\infty
$$

where

$$
\begin{aligned}
u^{(i)}(t)>0 & \text { for } i=0,1, \ldots, k \text { and } \\
(-1)^{i-k} u^{(i)}(t)>0 & \text { for } i=k+1, \ldots, n
\end{aligned}
$$

[7, p. 68]. Because of the above error, Nehari is led to the following false conclusions.

I. If $n$ is even and $p$ is positive either all solutions of (1) oscillate or none do.

II. If $n$ is even and $p$ is negative and (1) has an oscillatory solution then all positive nonoscillatory solutions are either strongly increasing or strongly decreasing, where a solution is said to be strongly increasing if $y^{(i)}(x)>0$ for $i=0, \ldots, n$, and is said to be strongly decreasing if $(-1)^{\dot{y}} y^{(i)}>0$ for $i=$ $0, \ldots, n$.

In §2, we will give necessary and sufficient conditions for (1) to be $(k, n-k)$ disconjugate, i.e., have no solution with a zero of order $k$ followed by a zero of order $n-k$. These results will then be applied to show that I and II are false.

Received by the editors March 16, 1978 and, in revised form, March 19, 1979.

AMS (MOS) subject classifications (1970). Primary 34C10.

Key words and phrases. Differential equations, disconjugate, oscillation. 
In §3, we will consider specific examples and show that $I$ is false for $n>4$ and that II is false for $n>6$, but true if $n=6$.

2. Conditions for $(k, n-k)$ disconjugacy. It is well known that (1) is $(k, n-k)$ disconjugate [5] if $n-k$ is even and $p$ is positive or if $n-k$ is odd and $p$ is negative. On the other hand, examples show that there exist solutions of equations of the type (1) with zeros of order $k$ followed by zeros of order $n-k$ for all $k<n$ provided $n-k$ is even and $p$ is negative or $n-k$ is odd and $p$ is positive. Nehari [7] and Elias [1] have observed that $(k, n-k)$ disconjugacy of (1) is related to the existence of certain monotone solutions of (1) in the following way.

THeOREM 1. If $n-k$ is even and $p$ is negative or if $n-k$ is odd and $p$ is positive then a necessary and sufficient condition for (1) to be $(k, n-k)$ disconjugate is the existence of a solution $y$ of (1) such that

$$
\begin{aligned}
y^{(j)}(x)>0 & \text { for } j=0, \ldots, k, \\
(-1)^{(j-k)} y^{(j)}(x)>0 & \text { for } j=k+1, \ldots, n .
\end{aligned}
$$

For the necessary part of the theorem see [7, p. 60], together with Theorem 4.3. For the sufficiency see [1, pp. 273-274].

Using Theorem 1 and techniques similar to those used in [2] and [6], we obtain the following theorem.

THEOREM 2. If $p>0$ and

$$
y^{(n-2 j)}+\left(x^{2 j} / 2 j !\right) p y=0
$$

has a solution $y$ such that $y^{(i)}>0$, for $i=0, \ldots, n-2 j-1$, then (1) is $(n-2 j-1,2 j+1)$ disconjugate.

Proof. Let $y$ be a solution of (2) such that $y^{(i)}>0, i=0, \ldots, n-2 j-1$. Then

$$
y^{n-2 j-1}(x)=y^{n-2 j-1}(a)+\int_{x}^{a} \frac{t^{2 j}}{2 j !} p(t) y(t) d t
$$

Since $\lim _{x \rightarrow \infty} y^{n-2 j-1}(x)$ exists and is positive, we have

$$
y^{n-2 j-1}(x)>\int_{x}^{\infty} \frac{t^{2 j}}{2 j !} p(t) y(t) d t>\int_{x}^{\infty} \frac{(t-x)^{2 j}}{2 j !} p(t) y(t) d t .
$$

Thus

$$
\begin{aligned}
y(x)> & y(b)+y^{\prime}(b)(x-b)+\cdots+\frac{y^{(n-2 j-2)}(b)(x-b)^{n-2 j-2}}{(n-2 j-2) !} \\
& +\int_{b}^{x} \frac{(x-t)^{n-2 j-2}}{(n-2 j-2) !} \int_{t}^{\infty} \frac{(s-t)^{2 j}}{(2 j) !} p(s) y(s) d s .
\end{aligned}
$$


By the Monotone Convergence Theorem there is a function $z>0$ satisfying

$$
\begin{aligned}
z(x)= & y(b)+y^{\prime}(b)(x-b)+\cdots+\frac{y^{n-2 j-2}(b)(x-b)^{n-2 j-2}}{(n-2 j-2) !} \\
& +\int_{b}^{x} \frac{(x-t)^{n-2 j-2}}{(n-2 j-2) !} \int_{t}^{\infty} \frac{(s-t)^{2 j}}{(2 j) !} p(s) y(s) d s d t .
\end{aligned}
$$

Differentiating, we have $z^{n}(x)=-p(x) z(x)$ and

$$
\begin{aligned}
z^{i}(x)>0 & \text { for } i=0, \ldots, n-2 j-1, \\
(-1)^{i-n+2 j+1} z^{i}(x)>0 & \text { for } i=n-2 j, \ldots, n .
\end{aligned}
$$

Thus by Theorem 1 , we have $(1)$ is $(n-2 j-1,2 j+1)$ disconjugate.

If $p$ is negative we obtain a similar result. Since its proof is basically the same as Theorem 2, it will be omitted.

THEOREM 3. If $p<0$ and

$$
y^{(n-2 j+1)}-\frac{x^{2 j-1}}{(2 j-1) !} p(x) y=0
$$

has a solution $y$ such that $y^{(i)}>0$ for $i=0, \ldots, n-2 j$, then (1) is (n$2 j, 2 j$ ) disconjugate.

It should be noted that Theorems 2 and 3 can also be obtained via [7, Theorem 5.1]. By repeatedly applying Theorems 2 and 3 sufficient conditions for $(k, n-k)$ disconjugacy of (1) are thus obtained by comparing (1) to either a second or third order equation.

Using techniques as in [6], the following necessary condition for $(k, n-k)$ disconjugacy will enable us to give counterexamples to I and II.

TheOREM 4. If (1) is $(j, n-j)$ disconjugate, where $n-j$ is even for $p$ negative and odd for $p$ positive, then

$$
w^{\prime \prime}+\left[\frac{1}{(n-j-2) !} \frac{1}{(j-1) !} \int_{t}^{\infty}(s-t)^{n-3}|p(s)| d s\right] w=0
$$

is nonoscillatory.

Proof. Suppose $(1)$ is $(j, n-j)$ disconjugate. Then there is a solution $y$ of (1) such that

$$
\begin{aligned}
y^{(i)}>0 & \text { for } i=0, \ldots, j, \\
(-1)^{i-j} y^{(i)}>0 & \text { for } i=j+1, \ldots, n .
\end{aligned}
$$

Since $y^{(i)}>0$ for $i=0, \ldots, j$ and $y^{i-1}$ is increasing, integrating $y^{j-1}$ from $a$ to $x j-1$ times, we obtain

$$
\begin{aligned}
y(x) & \geqslant \frac{1}{(j-2) !} \int_{a}^{x}(x-t)^{j-2} y^{j-1}(t) d t \geqslant \frac{y^{j-1}(a)}{(j-2) !} \int_{a}^{x}(x-t)^{j-2} d t \\
& =\frac{y^{j-1}(a)}{(j-1) !}(x-a)^{j-1} .
\end{aligned}
$$


Since $y^{j+1}<0$ and $\lim _{x \rightarrow \infty} y^{i}(x)=0$ for $i=j+1, \ldots, n$, we have

$$
\begin{aligned}
-y^{j+1}(x) & =\frac{1}{(n-j-2) !} \int_{x}^{\infty}(s-x)^{n-j-2}|p(s)| y(s) d s \\
& >\frac{1}{(n-j-2) !} \int_{x}^{\infty}(s-x)^{n-j-2}|p(s)| \frac{y^{j-1}(x)}{(j-1) !}(s-x)^{j-1} d s \\
& =\frac{y^{j-1}(x)}{(n-j-2) !} \frac{1}{(j-1) !} \int_{x}^{\infty}(s-x)^{n-3}|p(s)| d s .
\end{aligned}
$$

Letting $v=y^{j} / y^{j-1}$, we have

$$
v^{\prime}+v^{2} \leqslant \frac{-1}{(n-j-2) !} \frac{1}{(j-1) !} \int_{x}^{\infty}(s-x)^{n-3}|p(s)| d s
$$

and the result follows from a result of Wintner [8].

EXAMPLe 1. Consider

$$
y^{(10)}+\frac{k}{x^{10}} y=0 .
$$

By Theorem 4, (5) fails to be $(5,5)$ disconjugate provided

$$
w^{\prime \prime}+\left(\frac{1}{3 !} \frac{1}{4 !} \int_{t}^{\infty}(s-t)^{7} \frac{k}{s^{10}} d s\right) w=0
$$

is oscillatory, i.e., provided $k>2592$. Applying Theorem 2, (5) is $(9,1)$ disconjugate if

$$
w^{\prime \prime}+\left(x^{8} / 8 !\right)\left(k / x^{10}\right) w=0
$$

is nonoscillatory, which happens when $k<10,080$. Thus for $k=3000$, (5) is $(9,1)$ but not $(5,5)$ disconjugate. Hence, in view of the fact that nonoscillation of (1) implies eventual disconjugacy together with Theorem 1, we have a counterexample to I.

EXAMPLE 2. To show that II is false in general, consider

$$
y^{(12)}-\frac{k}{x^{12}} y=0 \text {. }
$$

Applying Theorem 4, we have that $(6)$ fails to be $(6,6)$ disconjugate if

$$
w^{\prime \prime}+\left[\frac{1}{4 !} \frac{1}{5 !} \int_{t}^{\infty}(s-t)^{9} \frac{k}{s^{12}} d s\right] w=0
$$

is oscillatory. But that happens when $k>79,200$. On the other hand (6) is $(2,10)$ disconjugate provided

$$
w^{\prime \prime \prime}+\frac{x^{9}}{9 !} \frac{k}{x^{12}} w=0
$$

is $(2,1)$ disconjugate. But that happens when $k<9$ ! $(2 / 3 \sqrt{3})<139,673$ [3]. Because of reasons as in Example 1, we have a counterexample to II if $k=80,000$. 
3. In this section we will consider specific examples to show that $I$ is false for $n>4$ and that II is false for $n>6$, but true for $n=6$.

Using ideas contained in [6] and [4], let $f(r)=-r(r-1)(r-2)(r-3)(r-$ $4)(r-5)$ and $g(r)=-(r+2)(r+1)(r)(r-1)(r-2)(r-3)$. Let

$$
A=\max \{f(r): 0 \leqslant r \leqslant 1\}
$$

and

$$
B=\max \{f(r): 2 \leqslant r \leqslant 3\}=\max \{g(r): 0 \leqslant r \leqslant 1\} .
$$

Since $(r+2)(r+1)<(r-4)(r-5)$, for $0 \leqslant r \leqslant 1$, we have $g(r)<f(r)$, for $0<r<1$. Since $g(r) \geqslant 0$ on $(0,1)$, it attains its maximum value at a point $r_{0}$ on the interior of the interval $(0,1)$. Thus

$$
B=g\left(r_{0}\right)<f\left(r_{0}\right) \leqslant A .
$$

Now choose $C$ such that $B<C<A$. Then

$$
f(r)-C=0
$$

has a real root between 0 and 1 , but has no real root in $[2,3]$. Thus it must have complex roots.

Letting $p(t)=C(t+1)^{-6}$, we see that $u(t)=(t+1)^{r}$ is a solution of

$$
y^{(6)}+p(t) y=0
$$

for $r$ any root of (7). Thus, since (7) has both real and complex roots, (8) must have both oscillatory and nonoscillatory solutions, contrary to $\mathrm{I}$.

The idea in the above example can be used to show that $I$ is false for $n>6$ and that II is false for $n \geqslant 8$. However, since the $\min \{f(r): 1 \leqslant r \leqslant 2\}=$ $\min \{f(r): 3 \leqslant r \leqslant 4\}$ we cannot give a counterexample to II for $n=6$. Indeed, II is true for $n=6$.

THEOREM 5. If

$$
y^{(6)}-p y=0
$$

with $p>0$ is oscillatory, then every nonoscillatory solution is either strongly increasing or strongly decreasing.

Proof. Because of the sign condition on $p$ any nonoscillatory solution other than a strongly increasing or strongly decreasing one will imply that (1) is either $(4,2)$ or $(2,4)$ disconjugate. But since $(9)$ is selfadjoint it is $(4,2)$ disconjugate if and only if it is $(2,4)$ disconjugate. Thus if there exists such a nonoscillatory solution it is disconjugate.

\section{REFERENCES}

1. Uri Elias, Nonoscillation and eventual disconjugacy, Proc. Amer. Math. Soc. 66 (1977), 269-275.

2. R. Grimmer, Comparison theorems for third and fourth order linear equations, J. Differential Equations 25 (1977), 1-10.

3. M. Hanan, Oscillation criteria for third-order linear differential equations, Pacific J. Math. 11 (1961), 919-944. 
4. W. J. Kim, On the zeros of solutions of $y^{(n)}+p y=0$, J. Math. Anal. Appl. 25 (1969), 189-208.

5. A. J. Levin, Some questions on the ascillation of solutions of a linear differential equation, Dokl. Akad. Nauk SSSR 148 (1963), 512-515.

6. David Lovelady, Oscillation and a class of odd order linear differential equations, Hiroshima J. Math. 5 (1975), 371-383.

7. Zeev Nehari, Green's function and disconjugacy. Arch. Rational Mech. Anal. 62 (1976), 53-76.

8. A. Wintner, On the nonexistence of conjugate points, Amer. J. Math. 73 (1951), 368-380.

Department of Mathematics, Murray State Universtty, Murray, Kentucky 42071 